\title{
FAMÍLIA SCENEDESMACEAE (CHLOROPHYCEAE, CHLOROCOCCALES) DO LAGO ÁGUA PRETA, MUNICÍPIO DE BELÉM, ESTADO DO PARÁ ${ }^{1}$
}

\author{
Regina Célia Viana Martins-Da-Silva ${ }^{2}$
}

Recebido em 4/3/97. Aceito em 3/12/97

\begin{abstract}
RESUMO - (Familia Scenedesmaceae (Chlorophyceae, Chlorococcales) do lago Água Preta, Municipio de Belém, Estado do Pará). Trata-se do inventário florístico da família Scenedesmaceae do lago Ȧgua Preta, parte do complexo de abastecimento de água do município de Belém, PA, efetuado a partir de quatro coletas, no periodo de outubro de 1992 a agosto de 1993. Os resultados mostraram que a familia Scenedesmaceae está representada por 15 táxons: Crucigenia fenestrata Schimidle, C. quadrata Morren, Dicloster acuatus Jao, Wei \& Hu, Dimorphococcus lunatus Braun, Scenedesmus acuminatus (Lagerheim) Chodat var. acuminatus, S. acuminatus (Lagerheim) Chodat var. bernardii (G.M. Smith) Dedussenko, S. armatus Chodat var. bicaudatus (Guglielmetti) Chodat, $S$. indicus Philipose, S. perforatus Lemmermann var. perforatus, S. quadricauda (Turpin) Brébisson var. quadricauda, Scenedesmus sp., Tetrallantos lagerheimii Teiling, Tetrastrum heteracanthum (Nordstedt) Chodat, T. punctatum (Schimidle) Ahlstrom \& Tiffany e Westella botryoides (W. West) De Wildeman.
\end{abstract}

Palavras-chave: algas, Amazônia, fitoplâncton, Chlorophyceae

\begin{abstract}
Scenedesmaceae Family (Chlorophyceae, Chlorococcales) from Água Preta Lake, Belém City, Pará State). An inventory of the Scenedesmaceae was made in lake Água Preta. This lake is the main component of the water reservoir of Belém, in Pará State. The inventory was based on four samples collected, during one year, from October 1992 to August 1993. The Scenedesmaceae family is represented by 15 taxa: Crucigenia fenestrata Schimidle, C. quadrata Morren, Dicloster acuatus Jao, Wei \& Hu, Dimorphococcus lunatus Braun, Scenedesmus acuminatus (Lagerheim) Chodat var. acuminatus, $S$. acuminatus (Lagerheim) Chodat var. bernardii (G.M. Smith) Dedussenko, S. armatus Chodat var. bicaudatus (Guglielmetti) Chodat, $S$. indicus Philipose, S. perforatus Lemmermann var. perforatus, $S$. quadricauda (Turpin) Brébisson var. quadricauda, Scenedesmus sp., Tetrallantos lagerheimii Teiling, Tetrastrum heteracanthum (Nordstedt) Chodat, T. punctatum (Schimidle) AhIstrom \& Tiffany and Westella botryoides (W. West) De Wildeman.
\end{abstract}

Key words: algae, Amazonia, fitoplankton, Chlorophyceae 


\section{Introdução}

O primeiro levantamento sobre a ordem Chlorococcales no Estado do Pará foi realizado por Kammerer (1938), que registrou 68 táxons infragenéricos. O segundo levantamento sobre a referida ordem no Estado, foi realizado por Martins-Da-Silva (1994), no qual foram relatados 46 táxons infragenéricos. Outros estudos realizados no Pará, que se referem a essa ordem porém não são restritos às Chlorococcales, estão representados por Grönblad (1945), Thomasson (1971; 1977), Uherkovich (1976; 1981) e Huszar (1994).

O Estado do Pará é muito rico em ambientes limnéticos e pouco, ainda, se conhece sobre suas algas. Este trabalho teve como objetivo o estudo da família Scenedesmaceae no lago Água Preta, visando contribuir para o conhecimento da ficoflórula dessa região.

\section{Material e métodos}

Área de estudo - Atualmente, a captação, o tratamento e a distribuição da água em Belém são efetuados pela Companhia de Saneamento do Pará (COSANPA), cuja represa é formada por dois grandes lagos, o Bolonha e o Água Preta, que ocupam, respectivamente, áreas de $1.790 .000 \mathrm{~m}^{2}$ e $7.199 .500 \mathrm{~m}^{2}$. O lago Água Preta possui capacidade de $10,55 \times 10^{6} \mathrm{~m}^{3}$, sendo o principal abastecedor de água que serve o município de Belém (COSANPA 1981).

Segundo Dias (1991), a área estudada localiza-se no quadrante a $48^{\circ} 11^{\prime} 00^{\prime \prime}$ e $48^{\circ} 13^{\prime} 48^{\prime \prime} \mathrm{W}$ e $1^{\circ} 21^{\prime} 32^{\prime}$ ' e $1^{\circ} 24^{\prime} 54^{\prime \prime}$ S, município de Belém, Estado do Pará. Este estudo foi desenvolvido no lago Água Preta, maior lago do complexo de abastecimento de água da cidade de Belém (Fig. 1).

O clima da região é do tipo Af, tropical chuvoso, com temperatura superior a $18^{\circ} \mathrm{C}$, sendo a média anual de $25,7^{\circ} \mathrm{C}$. A média dos meses mais quentes chega a $26,5^{\circ} \mathrm{C}$ e a dos meses mais frios, a $25^{\circ} \mathrm{C}$ (Köppen 1948 ).

O lago Água Preta encontra-se sobre sedimentos do Quaternário Antigo e Recente oriundos do Grupo Barreiras, o qual é formado por sedimentos arenosos e argilosos de origem continental (Moreira 1966; Ackermann 1969).

A cobertura vegetal é caracterizada por floresta densa de terra firme, floresta de terra firme aberta, floresta de várzea, capoeira de terra firme e áreas cultivadas (IDESP 1979).

Foram realizadas quatro coletas ao longo de um ano: em 28/outubro/1992, no início do período chuvoso; em 4/fevereiro/1993, quando as chuvas são abundantes; em 3/junho/1993, final da época chuvosa e em 30/agosto/1993, quando o índice pluviométrico apresenta-se baixo.

As amostras foram coletadas com rede de malha de $45 \mu \mathrm{m}$ de abertura. Foram também obtidas amostras de algas através de espremido de macrófitas do gênero Salvinia existentes no local. O metafiton foi coletado passando-se somente o recipiente de plástico da rede na massa d' água superficial. 


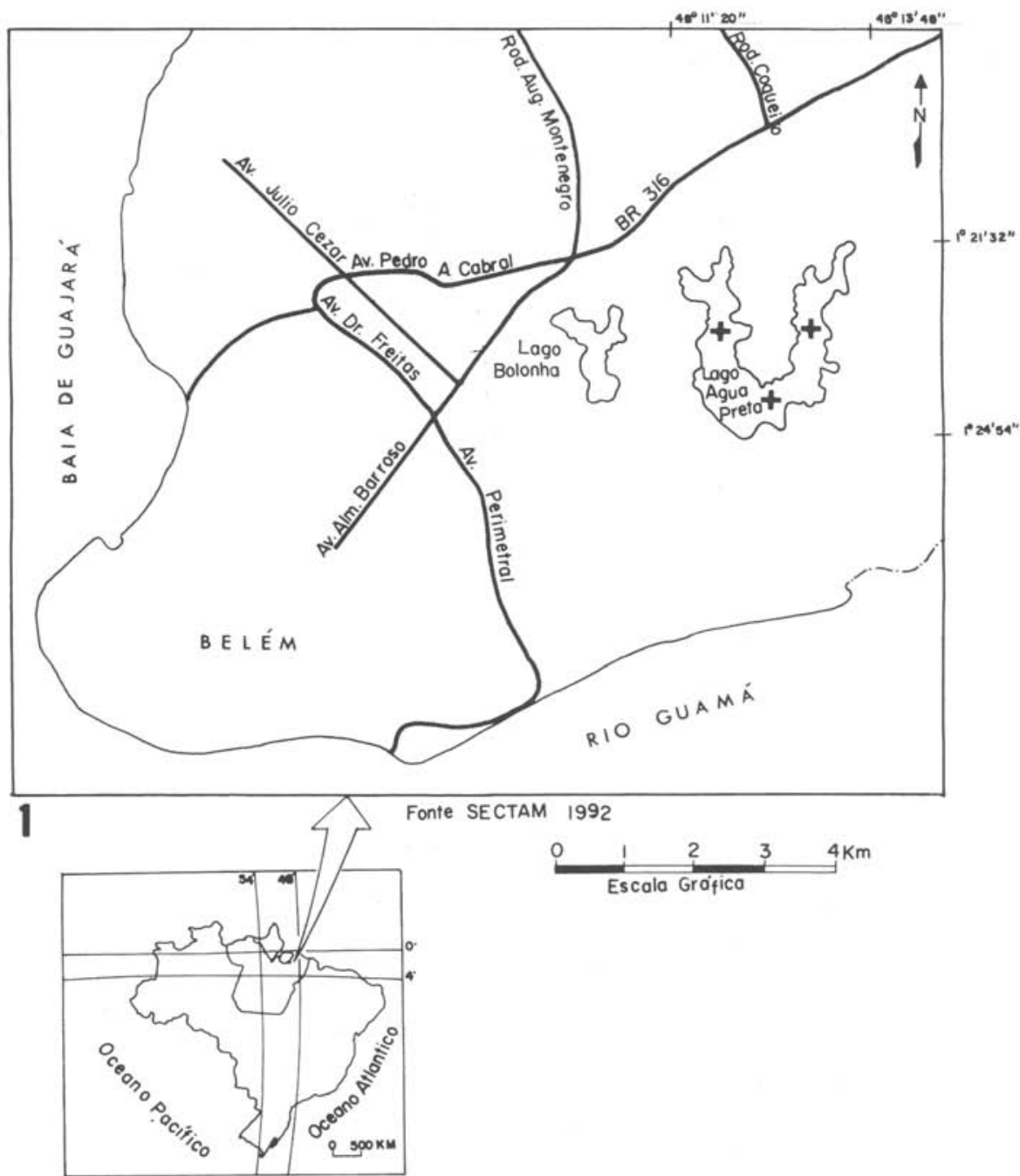

Figura1. Localização da área de estudo. Pontos de coleta (+) (adaptado de RADAMBRASIL 1976 e SECTAM 1992). 
O material recolhido foi tratado segundo Bicudo \& Bicudo (1970). As amostras referidas neste trabalho encontram-se depositadas no Herbário IAN, da Embrapa Amazônia Oriental.

De acordo com os dados obtidos durante as observações ao microscópio, mensurações e desenhos, foi realizada a identificação taxonômica, baseada em Kómarek \& Fott (1983), subsidiada por Kammerer (1938), Comas (1980; 1984), Tell \& Mosto (1982), Sant'Anna \& Martins (1982), Komárek (1983), Hindák (1984), Sant'Anna (1984) e Nogueira (1991). As formas celulares usadas na descrição das células foram padronizadas de acordo com Stearn (1983).

\section{Resultados e discussão}

Chave para as espécies e as variedades de Scenedesmaceae do Lago Água Preta

1. Cenóbio com células trapezoidais sem pirenóide 1. Crucigenia fenestrata

1. Cenóbio com células de formato diferente, com pirenóides

2. Cenóbio com células lunadas apresentando ápices em agulha, com dois pirenóides

2. Cenóbio com células lunadas ou diferentes, com um pirenóide

3. Cenóbio arredondado, células ligadas por fios mucilaginosos 4. Dimorphococcus lunatus

3. Cenóbio arredondado ou diferente, células não ligadas por fios mucilaginosos

4. Cenóbio arredondado a alongado, células reniformes.

12. Tetrallantos lagerheimii

4. Cenóbio arredondado a alongado ou diferente, células não reniformes

5. Cenóbio arredondado a alongado, células esféricas, presença de bainha mucilaginosa. 15. Westella botryoides

5. Cenóbio diferente, células não esféricas, ausência de bainha mucilaginosa

6. Cenóbio quadrático, células triangulares com dois espinhos retos na margem externa 13. Tetrastrum heteracanthum

6. Cenóbio quadrático ou diferente, células triangulares ou diferentes sem espinhos ou quando presentes, nos pólos

7. Cenóbio quadrático, células triangulares, espinhos ausentes, espaço intercelular retangular estreito

7. Cenóbio quadrático ou diferente, células não triangulares, com ou sem espaço intercelular, espinhos ausentes, quando presentes, nos pólos

8. Cenóbio quadrático, células oblongas, espaço intercelular quadrático 2. Crucigenia quadratica

8. Cenóbio diferente, células oblongas ou diferentes, sem espaço intercelular

9. Cenóbio reto, linear, células oblongas, um espinho reto nas células externas, dispostos em diagonal ......................... 7. Scenedesmus armatus var. bicaudatus

9. Cenóbio reto, linear ou em ziguezague, células oblongas ou diferentes, dois espinhos curvos ou ausentes nas células externas

10. Cenóbio reto, linear, clatrado, com dois espinhos nas células externas 
10. Cenóbio reto, linear ou em ziguezague, não clatrado, com dois ou mais espinhos ou ausentes

11. Cenóbio reto, linear, células externas com dois espinhos e as internas com um espinho

11. Cenóbio reto, linear ou em ziguezague, células externas com ou sem espinhos e as internas sem espinho

12. Cenóbio reto, linear, células externas com dois espinhos e as internas sem espinhos ou com vários

10. S. quadricauda var. quadricauda

12. Cenóbio reto, linear ou em ziguezague, espinhos ausentes

13. Cenóbio reto, linear, células externas lunadas, internas fusiformes

5. S. acuminatus var. acuminatus

13. Cenóbio reto, em ziguezague, todas as células lunadas ou cilíndricas

14. Cenóbio reto, em ziguezague, células lunadas

6. $S$. acuminatus var. bernardii

14. Cenóbio reto, em ziguezague, células cilíndricas.

8. S. indicus

Descrição das espécies e das variedades

1. Crucigenia fenestrata (Schmidle) Schmidle. Allg. Bot. Z. 6: 234. 1900.

Fig. 2.

Basiônimo: Staurogenia fenestrata Schmidle. Allg. Bot. Z. 3: 107, f. 1. 1897.

Cenóbio quadrático; 4 células trapezoidais; $2-4 \times 5-8,5 \mu \mathrm{m}$; dispostas cruciadamente; lado mais estreito ligeiramente convexo voltado para o interior do cenóbio; margem externa côncava, quase reta; margem lateral retilínea; espaço intercelular quadrático; cloroplastídio único, parietal, sem pirenóide.

Amostras examinadas: BRASIL. Pará: Belém, lago Água Preta, 28/X/92, MartinsDa-Silva A01 (IAN); 04/II/93, Martins-Da-Silva A02 (IAN); 03/VI/93, MartinsDa-Silva \& Paiva A03 (IAN); 30/08/93, Martins-Da-Silva \& Paiva A04 (IAN).

Distribuição geográfica no Estado do Pará: primeiro registro da ocorrência da espécie.

Comentários: o gênero Crucigenia Morren, segundo Komárek \& Fott (1983), caracteriza-se por ter cenóbio quadrático, com espaço intercelular quadrado e células trapezoidais ou oblongas. Crucigenia fenestrata separa-se das demais espécies por apresentar lado externo das células reto ou ligeiramente côncavo e não possuir pirenóides. C. fenestrata diferencia-se de C. tetrapedia (Kirchner) West \& West por apresentar espaço intercelular relativamente largo, quadrado e ter lados côncavos, enquanto que a segunda apresenta o espaço entre as células muito pequeno, retangular ou ausente (Hindák 1984).

2. Crucigenia quadrata Morren. An. des Sc. Nat. 204: 415, pl. 15, f. 2-5. 1830. Fig. 3. 
Cenóbio quadrático; 4 células oblongas; 6,4-7,2×3,7-4,4mm, dispostas cruciadamente, comprimidas pelos ápices, espaço intercelular quadrático 4,6-5,1 $\mu \mathrm{m}$ larg.; cloroplastídio único, parietal, 1 pirenóide.

Amostras examinadas: BRASIL. Pará: Belém, lago Água Preta, 03/VI/93 MartinsDa-Silva \& Paiva A03 (IAN); 30/08/93, Martins-Da-Silva \& Paiva A04 (IAN).

Distribuição geográfica no Estado do Pará: Fazenda Taperinha, Santarém (Kammerer 1938).

Comentários: Crucigenia quadrata aproxima-se de C. mucronata (G.M. Smith) Komárek por ambas terem paredes convexas, espaço intercelular quadrático e a presença de pirenóide; porém difere quanto à ornamentação da parede celular, cujas regiões tangenciais na segunda espécie apresentam duas projeções em formato de verrugas, enquanto que na primeira, as paredes exibem-se completamente lisas. Das demais espécies, C. quadrata se diferencia por apresentar pirenóides (Komárek 1974).

3. Dicloster acuatus Jao, Wei \& Hu. Acta Hydrobiol. Sinica 6 (1): 115, f. 1-7. 1976. Fig. 4-5.

Cenóbio com 1 ou 2 conjuntos de 2 células lunadas; ápices afilados abruptamente, alongando-se em forma de agulha; 2,8-5,0 $\mu \mathrm{m}$ larg.; distância entre os ápices 43$58 \mu \mathrm{m}$; unidas pela parte convexa; cloroplastídio único, parietal, 2 pirenóides.

Amostras examinadas: BRASIL. Pará: Belém, lago Água Preta, 28/X/92, MartinsDa-Silva A01 (IAN); 04/II/93, Martins-Da-Silva A02 (IAN).

Distribuição geográfica no Estado do Pará: primeiro registro da ocorrência do gênero.

Comentários: Dicloster acuatus e Scenedesmus acuminatus (Lagerheim) Chodat são duas espécies muito próximas que se separam pelo número de pirenóides na célula; a primeira apresenta dois pirenóides e a segunda, apenas um. Esta é uma espécie pouco encontrada em literatura, seu primeiro registro para o Brasil foi feito por Nogueira (1991) ao analisar material do Rio de Janeiro. Assim como os representantes encontrados pela última autora, os que foram estudados neste trabalho apresentaram células mais curvadas que outras; mas não foram encontrados indivíduos com ápices arredondados, como foi registrado por Nogueira (1991).

4. Dimorphococcus lunatus A. Braun, Alg. unicel. gen. nov. min. cognita: 44. 1855. Fig. 6.

Cenóbio arredondado; grupos de 4 células unidas por um fio mucilaginoso ramificado, dispostas alternadamente; as 2 centrais são elípticas e as 2 externas reniformes a cordiformes; células com 12-18 x8-11 $\mu \mathrm{m}$; cloroplastídio único, parietal, 1 pirenóide.

Amostra examinada: BRASIL. Pará: Belém, lago Água Preta, 28/X/92, MartinsDa-Silva A01 (IAN). 
Distribuição geográfica no Estado do Pará: Fazenda Taperinha, Santarém (Kammerer 1938; Grönblad 1945); lagos Jurucuí e Maicá, rio Tapajós (Thomasson 1971); rio Tapajós (Uherkovich 1976); região de Nhamundá, Terra Santa (Thomasson 1977); baía do Guajará (Paiva 1991).

Comentários: a presença de fios mucilaginosos interligando grupos de células poderia sugerir uma semelhança com Dictyosphaerium Nägeli que logo é descartada quando se leva em consideração a morfologia das células e o arranjo no cenóbio.

5. Scenedesmus acuminatus (Lagerheim) Chodat var. acuminatus, Beitrage Kryptog. Sch.: 211, f. 88. 1902.

Fig. 7 .

Basiônimo: Selenastrum acuminatum Lagerheim, Öf. af K. Vetensk. Förh. 39 (2): 71 , pl. 3 , f. 27-30. 1882 .

Cenóbio reto, linear; 4-8 células, as externas lunadas, ápices agudos voltados para o exterior do cenóbio; as internas fusiformes, retas, ápices agudos; 19-31x4$6 \mu \mathrm{m}$; parede celular lisa; cloroplastídio único, parietal, 1 pirenóide.

Amostras examinadas: BRASIL. Pará: Belém, lago Água Preta, 28/X/92, MartinsDa-Silva A01 (IAN); 04/II/93, Martins-Da-Silva A02 (IAN); 03/VI/93, Martins-DaSilva \& Paiva A03 (IAN); 30/08/93, Martins-Da-Silva \& Paiva A04 (IAN).

Distribuição geográfica no Estado do Pará: primeiro registro da ocorrência da espécie.

Comentários: optou-se por considerar os espécimes estudados como Scenedesmus acuminatus (Lagerheim) Chodat, de acordo com Uherkovich (1966), Komárek \& Fott (1983) e Hindák (1990) pois estes autores consideraram $S$. falcatus Chodat como sinônimo de $S$. acuminatus.

Hegewald et al. (1990) colocaram Scenedesmus falcatus como sinônimo de $S$. pectinatus Meyen, o qual já havia sido considerado como sinônimo de $S$. acutus Meyen, por Uherkovich (1966). Hindák (1990) considerou este último como sinônimo de $S$. obliquus (Turpin) Kützing. A sistemática de Scenedesmus é, realmente, muito complexa. Nogueira (1991), estudando trinta e sete táxons do referido gênero, fez um histórico a respeito da problemática taxonômica de $S$. acuminatus.

6. Scenedesmus acuminatus (Lagerheim) Chodat var. bernardii (G. M. Smith) Dedussenko. Korsikov, Protococcineae 380, f. 368. 1953.

Fig. 8-9.

Cenóbio reto, em ziguezague; 4-8 células, lunadas, extremidades agudas; $25-$ $32 \times 2,5-4 \mu \mathrm{m}$; metade das células arranjadas com a parte côncava voltada para a direita e as outras com essa região voltada para a esquerda; pólo de uma célula se prende à região mediana convexa da vizinha; as duas centrais se prendem parte convexa com parte convexa; cloroplastídio único, parietal, 1 pirenóide. 

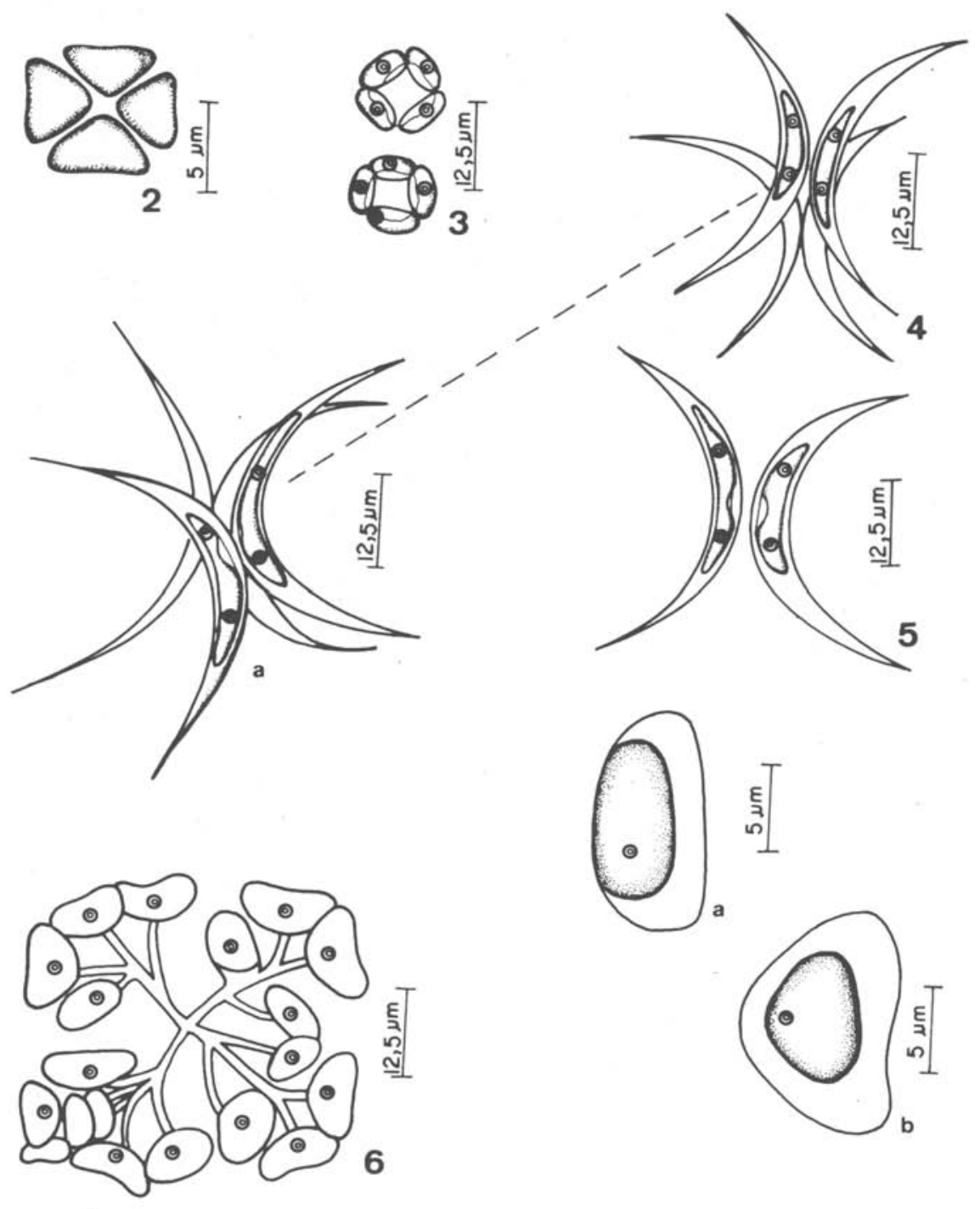

Figura 2. Crucigenia fenestrata (Schmidle) Schmidle. Figura 3. Crucigenia quadrata Morren. Figura 4-5. Dicloster acuatus Jao, Wei \& Hu ; 4 e 4a, mesmo cenóbio em diferentes posições. Figura 6. Dimorphococus lunatus A. Braun; a. uma célula interna do cenóbio; b. uma célula externa do cenóbio. 
Amostras examinadas: BRASIL. Pará: Belém, lago Água Preta, 28/X/92, MartinsDa-Silva A01 (IAN); 04/II/93, Martins-Da-Silva A02 (IAN); 03/VI/93, Martins-DaSilva \& Paiva A03 (IAN).

Distribuição geográfica no Estado do Pará: primeiro registro da ocorrência da espécie.

Comentários: Scenedesmus acuminatus var. bernardii é um táxon que ainda gera certa controvérsia. Uherkovich (1966) confirmou $S$. bernardii como sinônimo de $S$. acuminatus var. bernardi. Komárek \& Fott (1983) refereriram-se ao táxon em questão como $S$. bernardii, e não como uma variedade de acuminatus. Sant'Anna (1984) concordou com Uherkovich (1966), enquanto que Nogueira (1991) referiu-se a Scenedesmus bernardii como espécie e não como variedade.

Optou-se por identificar os espécimes estudados de acordo com Uherkovich (1966), por ser o trabalho mais recente de revisão que trata da posição do táxon em questão, até que novos estudos venham a esclarecer em definitivo a posição taxonômica correta dessas algas.

Nas amostras examinadas, as células apresentaram-se com o comprimento variando entre 26-33 $\mu \mathrm{m}$, enquanto Uherkovich (1966) apresenta os limites métricos

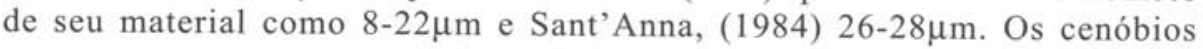
apresentaram-se ora com as células mais largas, como na Fig. 9, e ora mais estreitas, como na Fig. 8.

7. Scenedesmus armatus Chodat var. bicaudatus (Guglielmetti) Chodat. Z. Hydrol. 3: 204, f. 106.1926.

Fig. 10 .

Basiônimo: Scenedesmus acutiformis Schröder var. bicaudatus Guglielmetti. N. Not. 21: 31. 1910.

Cenóbio reto, linear; 4 células oblongas; $8-12 \times 2,5-4 \mu \mathrm{m}$; as células externas levemente arqueadas para o interior do cenóbio; com um espinho reto em um dos pólos, disposto em diagonal e três dentes no outro pólo; as internas, com costelas longitudinais dispostas em ambas as faces, interrompidas na parte mediana; cloroplastídio único, parietal, 1 pirenóide.

Amostras examinadas: BRASIL. Pará: Belém, lago Água Preta, 28/X/92, MartinsDa-Silva A01 (IAN); 04/II/93, Martins-Da-Silva A02 (IAN). 03/VI/93, Martins-DaSilva \& Paiva A03 (IAN); 30/08/93, Martins-Da-Silva \& Paiva A04 (IAN).

Distribuição geográfica no Estado do Pará: primeiro registro da ocorrência da variedade.

Comentários: segundo Uherkovich (1966), a característica que separa Scenedesmus armatus da espécie mais próxima, S. semipulcher Hortobágyi, é a presença de costelas em ambas as faces do primeiro táxon. A população estudada foi identificada como $S$. 
armatus var. bicaudatus por ter as costelas em ambas as faces e ser bicaudada, sendo esta última característica a que separa o táxon dos demais infra-específicos.

Komárek \& Fott (1983) transferiram S. armatus var. bicaudatus para $S$. semipulcher. Hindák (1990) reuniu os táxons infragenéricos de S. armatus ao nível de espécie, por achar que esse é um grupo de Scenedesmus, taxonomicamente, ainda confuso devido a grande quantidade de táxons propostos e por não ter dados, a partir desse seu estudo, capazes de servirem como fronteiras limitantes entre as variedades. Hegewald et al. (1990) estudando Scenedesmus do Sul da Índia, separaram S. armatus em variedades; $S$. armatus var. bicaudatus foi reconhecido à parte das demais pelo caráter bicaudado. Desta forma, a identificação, no presente estudo, está de acordo com Uherkovich (1966) e Hegewald et al. (1990).

8. Scenedesmus indicus Philipose. Chlorococcales, India Council of Agric. Res. 258. 1967.

Fig. 11-12.

Cenóbio reto, em ziguezague, mucilagem presente; 2-4 células cilíndricas, arqueadas, $12-15,5 \times 4,5-7,5 \mu \mathrm{m}$, pólos alargados em forma de chapéu; dispõem-se com a região côncava voltada para a periferia; as internas prendem-se à região mediana das externas através de seus pólos; cloroplastídio único, parietal, 1 pirenóide.

Amostras examinadas: BRASIL. Pará: Belém, lago Água Preta, 28/X/92, MartinsDa-Silva A01 (IAN); 04/II/93, Martins-Da-Silva A02 (IAN); 03/VI/93, Martins-DaSilva \& Paiva A03 (IAN); 30/08/93, Martins-Da-Silva \& Paiva A04 (IAN).

Distribuição geográfica no Estado do Pará: primeiro registro da ocorrência da espécie.

Comentários: Scenedesmus indicus é uma espécie que se diferencia das demais, com certa facilidade, devido à morfologia de suas células e seu arranjo no cenóbio. $S$. indicus lembra $S$. productocapitatus Schmula e Pseudotetradesmus quaternarius Hirose \& Ariyama pela forma de suas células, mas diferencia-se das duas espécies citadas por ter o cenóbio com células em ziguezague, enquanto as duas últimas apresentam cenóbios lineares.

9. Scenedesmus perforatus Lemmermann var. perforatus. Z. Fisch., 11(2): 104 f. 3.1903. Fig. 13.

Cenóbio reto, linear, clatrado; 4 células oblongas, $22-34 \times 6-11 \mu \mathrm{m}$; unidas longitudinalmente na região polar; as externas com margem externa ligeiramente convexa e margem interna côncava; pólos arredondados, 1 espinho curvo em cada pólo; as internas com ambas as margens côncavas na região central; cloroplastídio único, parietal, 1 pirenóide.

Amostras examinadas: BRASIL, Pará: Belém, lago Água Preta, 28/X/92, MartinsDa-Silva A01 (IAN); 03/VI/93, Martins-Da-Silva \& Paiva A03 (IAN). 
Distribuição geográfica no Estado do Pará: Fazenda Taperinha, Santarém (Kammerer 1938); rio Tapajós, Santarém (Uherkovich 1976); baía do Guajará (Paiva 1991).

Comentários: Scenedesmus perforatus var. perforatus é um táxon que não oferece barreiras na sua identificação. O cenóbio clatrado e a forma das células separam a espécie das demais do gênero. Aproximando-se, ainda, de $S$. tropicus Crow, do qual difere pela forma das células e a maneira como estas se unem para formar o cenóbio; na primeira, as células são elípticas a oblongas e ligam-se apenas pelo toque de suas paredes; e em $S$. tropicus, as células são elípticas alongadas e se unem através de projeções da parede celular de uma célula em direção à outra, em dois pontos próximos às regiões polares.

Hegewald et al. (1990) consideraram a espécie em questão como um dos táxons de Scenedesmus que apresenta menos variabilidade e chamaram a atenção para o espaço intercelular, quando as células entram em processo de divisão, pois estas tornamse mais amplas, reduzindo, conseqüentemente, o espaço entre as mesmas.

Nogueira (1991), estudando material do Rio de Janeiro, encontrou indivíduos com apenas um ou dois espaços intercelulares no cenóbio; esta característica encaixase no alerta feito por Hegewald et al. (1990).

10. Scenedesmus quadricauda (Turpin) Brébisson var. quadricauda. In: Brébisson \& Godey, Mém. Soc. Acad. 1: 66. 1835.

Fig. 14-18.

Basiônimo: Achnanthes quadicauda Turpin, In: Dic. sc. nat. Pl. bot. Vég. acot., p.14, f. 13. 1820 .

Cenóbio reto, linear; 4-8 células oblongas; 9-23×2,5-10 $\mu \mathrm{m}$; pólos arredondados; margem externa, das células externas, retas à ligeiramente convexas, 1 espinho (2,3$20 \mu \mathrm{m}$ ) curvo em cada pólo disposto diagonalmente; cloroplastídio único, parietal, 1 pirenóide.

Amostras examinadas: BRASIL. Pará: Belém, lago Água Preta, 28/X/92, MartinsDa-Silva A01 (IAN); 04/II/93, Martins-Da-Silva A02 (IAN); 03/VI/93, Martins-DaSilva \& Paiva A03 (IAN); 30/08/93, Martins-Da-Silva \& Paiva A04 (IAN).

Distribuição geográfica no Estado do Pará: Fazenda Taperinha, Santarém (Kammerer 1938); rio Tapajós, Santarém (Uherkovich 1976); região de Nhamundá, Terra Santa (Thomasson 1977).

Comentários: A diferença entre Scenedesmus quadricauda var. quadricauda e $S$. quadricauda (Turpin) Bréb. var. maximus West \& West baseia-se, apenas, nas dimensões. Quando os táxons da variedade típica se apresentam com os valores máximos e os de $S$. quadricauda var. maximus com valores mínimos, fica difícil identificá-los.

Komárek \& Ruzicka (1969) investigaram o efeito da temperatura sobre o crescimento e a variabilidade de $S$. quadricauda; dentre as suas conclusões, afirmaram que a dimensão das células aumenta com a elevação da intensidade luminosa à 

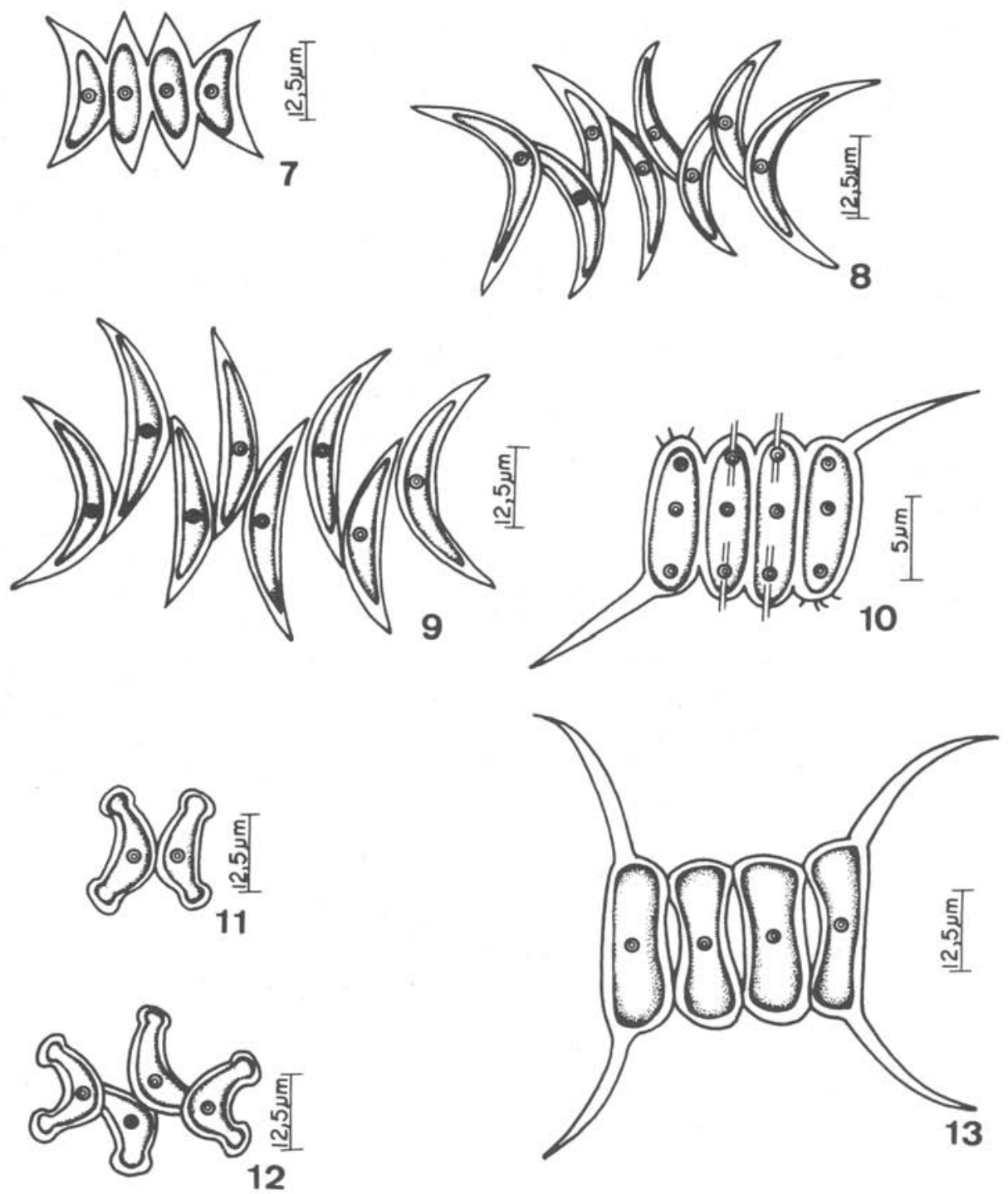

Figura 7. Scenedesmus acuminatus (Lag.) Chodat var. acuminatus. Figura 8-9. S. acuminatus (Lag.) Chodat var. bernardii (G. M. Smith) Ded. Figura 10. S. armatus Chodat var. bicaudatus (Gug.) Chodat. Figura 1112. S. indicus Phil. Figura 13. S. perforatus Lemm. var. perforatus. 
temperatura constante; e que à luminosidade constante e com o aumento da temperatura, o volume do cenóbio diminui.

As populações encontradas neste estudo apresentaram suas dimensões se sobrepondo aos limites entre as duas variedades. Por este motivo, por acreditar-se na grande capacidade de polimorfismo em Scemedesmus quadricauda e baseando-se nos resultados dos estudos de Komárek \& Ruzicka (1969), visto que o presente estudo foi realizado em ambiente tropical, onde a luminosidade solar e a temperatura são elevados, optou-se por restringir as populações estudadas à variedade típica. Em alguns cenóbios, usando-se imersão, foram observadas estruturas finas e delicadas como se fossem pequenos fios distribuidos nas paredes célulares.

\section{Scenesdesmus sp.}

Fig. 19.

Cenóbio reto, linear; 4 células oblongas a elípticas; $26-28,5 \times 7-10 \mu \mathrm{m}$; pólos arredondados; parede celular pontuada e provida de diminutas projeções filiformes; as externas com margem externa convexa e 1 espinho curvo 24-26 $\mu \mathrm{m}$, em cada polo; internas com um dos pólos com 1 espinho e o outro com 3 dentes dispostos diagonalmente em cada metade do cenóbio; cloroplastídio único, parietal, 1 pirenóide.

Amostra examinada: BRASIL. Pará: Belém, lago Água Preta, 28/X/92, MartinsDa-Silva A01 (IAN).

Comentários: os indivíduos analisados assemelham-se a Scenedesmus quadricauda (Turpin) Bréb. var. biornatus Kiss f. giganticus Uherkovich (Uherkovich 1966) a qual foi considerada, por Komárek \& Fott (1983), como sinônimo de S. magnus Meyen; difere deste pela presença, em Scenedesmus sp., das ornamentações na parede celular (pontuações e diminutas projeções cilíndricas, espinhos e dentes nos pólos das células internas). Faz-se necessário um estudo mais minucioso, para que se possa chegar a uma correta posição taxonômica, já que os representantes foram raros na área de estudo.

12. Tetrallantos lagerheimii Teil. S. Bot. Tid., 10: 62. 1916. Fig. 20.

Cenóbio arredondado a alongado com 4 células reniformes; 10-13,5x4-5,5 $\mu \mathrm{m}$; 2 têm ápices unidos e ocupam o mesmo plano, 2 se dispõem perpendicularmente e unem-se a um dos pólos das primeiras; cloroplastídio único, parietal, 1 pirenóide.

Amostras examinadas: BRASIL. Pará: Belém, lago Água Preta, 28/X/92, MartinsDa-Silva A01 (IAN); 04/II/93, Martins-Da-Silva A02 (IAN). 03/VI/93, Martins-DaSilva \& Paiva A03 (IAN); 30/08/93, Martins-Da-Silva \& Paiva A04 (IAN).

Distribuição geográfica no Estado do Pará: Lago Maicá, Santarém (Thomasson 1971).

Comentários: é um gênero monoespecifico e bem delimitado. A forma das células poderia sugerir outros gêneros, mas a disposição destas no cenóbio caracteriza-o completamente, separando-o em definitivo dos demais. 

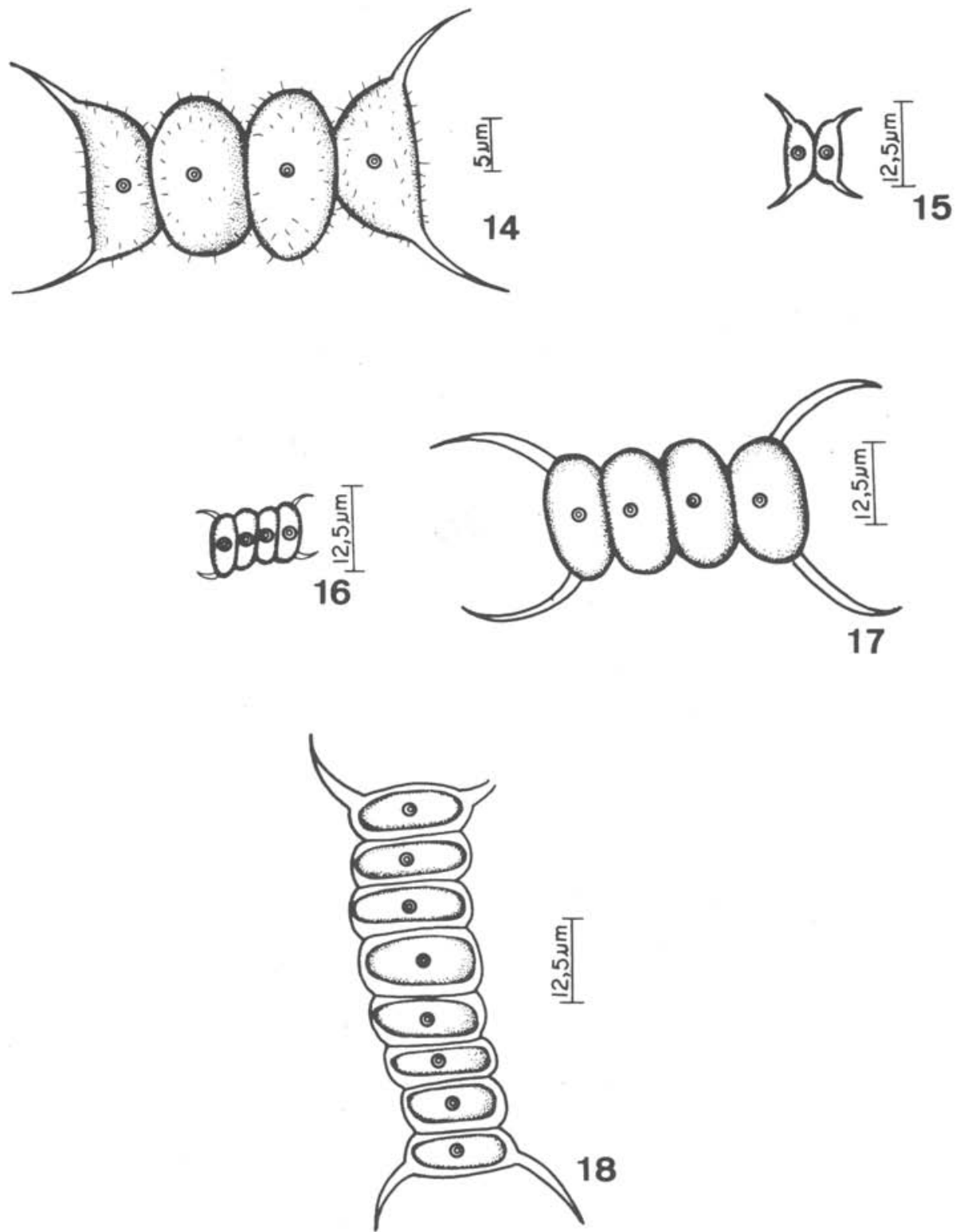

Figura 14-18. Scenedesmus quadricauda (Turpin) Bréb. var. quadricauda. 
13. Tetrastrum heteracanthum (Nordstedt) Chodat. Bull. Herb. Bois. 3: 113. 1895. Fig. 21.

Basiônimo: Staurogenia heteracantha Nordstedt In Wittrock \& Nordstedt., Bot. Not. 1882.

Cenóbio quadrático, cruciado; 4 células triangulares; 2,5-5 $\mu \mathrm{m}$ alt.; ângulos arredondados; 2 espinhos, finos, retos, de diferentes dimensões (maior 7-9 $\mu \mathrm{m}$, menor $2-3 \mu \mathrm{m})$, inseridos em margem externa da célula; espaço intercelular retangular; cloroplastídio único, parietal, 1 pirenóide.

Amostra examinada: BRASIL. Pará: Belém, lago Água Preta, 28/X/92, MartinsDa-Silva A01 (IAN).

Distribuição geográfica no Estado do Pará: primeiro registro da ocorrência da espécie.

Comentário: Tetrastrum heteracanthum tem na forma, na disposição de suas células e também na presença e local de inserção dos espinhos, as características que facilitam a sua identificação. Entretanto, poderia ser confundido com T. homoiacanthum (HuberPestalozzi) Comas, do qual difere pelas dimensões dos espinhos, pois o último os tem em tamanhos iguais, enquanto que em T. heteracanthum essas estruturas são heterométricas.

14. Tetrastrum punctatum (Schmidle) Ahlstrom \& Tiffany. Am. J. Bot. 21: 504. 1934.

Fig. 22.

Cenóbio quadrático, cruciado; 4 células triangulares; 6-7,5 $\mu \mathrm{m}$ alt.; ângulos arredondados; parede celular provida com diminutas verrugas; espaço intercelular retangular estreito; cloroplastídio único, parietal, 1 pirenóide.

Amostra examinada: BRASIL. Pará: Belém, lago Água Preta, 28/X/92, MartinsDa-Silva $A 01$ (IAN).

Distribuição geográfica no Estado do Pará: primeiro registro da ocorrência da espécie.

Comentário: Tetrastrum punctatum difere das demais espécies do gênero por apresentar a parede celular ornada por estruturas em forma de verrugas.

15. Westella botryoides (W.West) De Wildeman. Bul. Herb. Bois., sér. 1, 5(6): 532. 1897.

Fig. 23.

Basionimo: Tetracoccus botryoides W. West., J. R. Mic. Soc. 2: 735. 1892.

Cenóbio arredondado a levemente alongado, cruciado, bainha de mucilagem presente; 4 células esféricas; 5-12 $\mu \mathrm{m}$ diâm.; espaço intercelular retangular reduzido; cloroplastídio único, poculiforme, 1 pirenóide. 

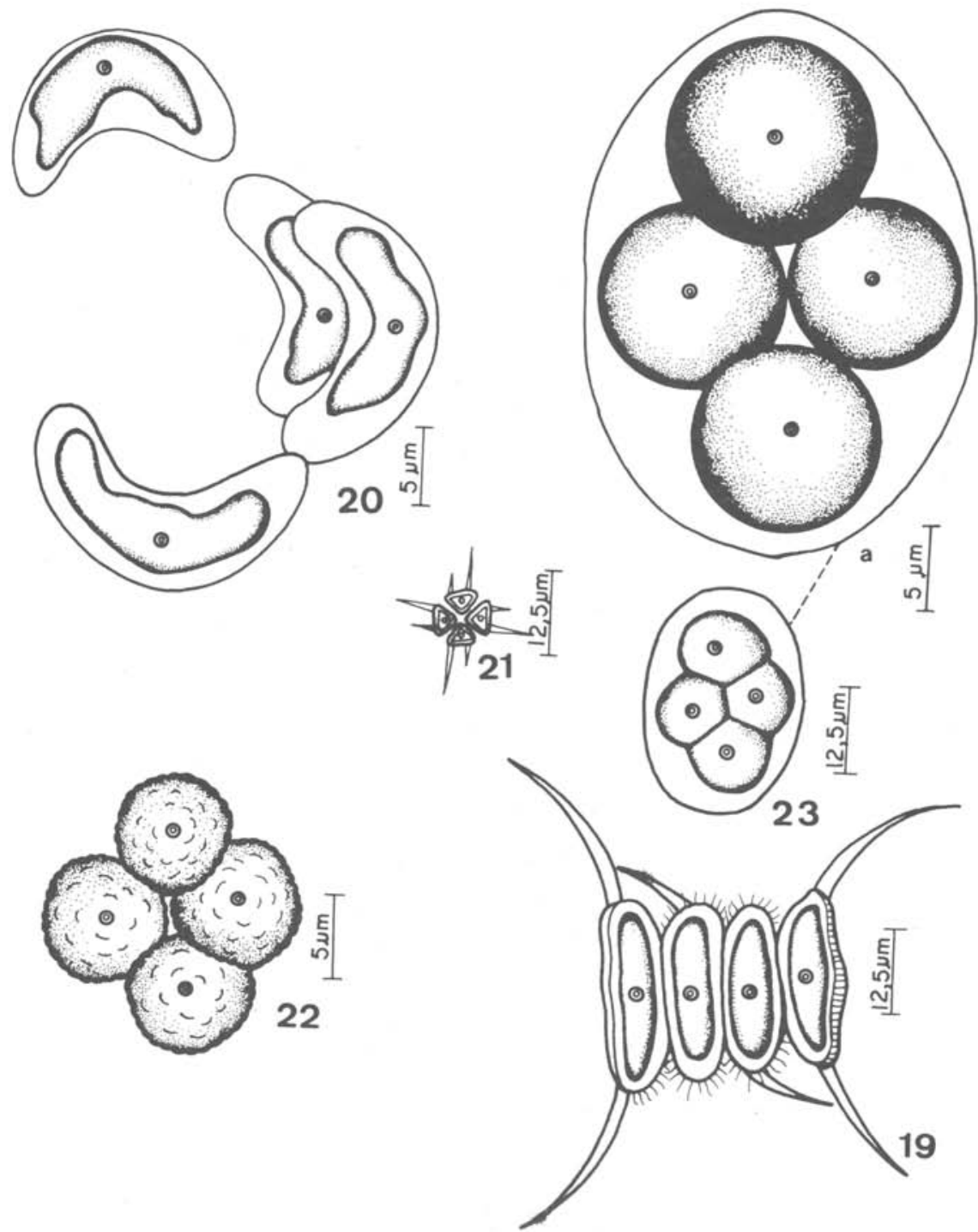

Figura 19. Scenedesmus sp. Figura 20. Tetrallantos lagerheimii Teil. Figura 21. Tetrastrum heteracanthum (Nordstedt) Chodat. Figura 22. T. punctatum (Schmidle) Ahlstrom \& Tiff. Figura 23. Westella botryoides (W. West) De-Wild.; a. cenóbio em maior aumento 
Amostras examinadas: BRASIL. Pará: Belém, lago Água Preta, 03/VI/93 MartinsDa-Silva \& Paiva A03 (IAN); 30/VIII/93, Martins-Da-Silva \& Paiva A04 (IAN).

Distribuição geográfica no Estado do Pará: Fazenda Taperinha, Santarém (Kammerer 1938)

Comentário: Westella De-Wild. possui uma única espécie que se caracteriza pelo arranjo cruciado de suas quatro células esféricas, formando um espaço intercelular, e pelo processo de liberação dos autósporos (Komárek 1974). Esta lembra Tetrastrum devido ao arranjo de suas células e ao espaço intercelular retangular. Porém a forma das células, totalmente esféricas, fizeram com que os espécimes estudados fossem identificados como Westella, apesar de não terem sido observados os freqüentes sincenóbios citados na literatura.

\section{Agradecimentos}

Ao Dr. Carlos Eduardo de Mattos Bicudo (Instituto de Botânica de São Paulo) e à Dra. Ermelinda Maria De-Lamonica-Freire (Universidade Federal de Mato Grosso) pela orientação durante a execução da Dissertação de Mestrado. À M. Sc. Ina de Souza Nogueira (Universidade Federal de Goiás), pela leitura crítica da parte taxonômica da referida Dissertação.

\section{Referências bibliográficas}

Ackermann, F.L. 1969. Esboço para a geologia entre a cidade de Belém-Rio Gurupi e Atlântico - Rio Guamá. Belém. Imprensa Universitária do Pará.

Bicudo, C.E. M.\& Bicudo, R.M.T. 1970. Algas de águas continentais brasileiras. São Paulo. Fundação Brasileira para o Desenvolvimento do Ensino de Ciências.

Comas, A. 1980. Nuevas e interesantes Chlorococcales (Chlorophyceae) de Cuba. Acta Botanica Cubana 2:1-18.

Comas, A. 1984. Chlorococcales (Chlorophyceae) de algunos acuatorios de Pinar del Rio, Cuba. Acta Botanica Cubana 17: 1-75.

COSANPA.1981. Diagnóstico do estado urbanístico e proteção sanitária dos lagos Bolonha e Água Preta, I. Belém. Sociedade Técnica de Engenharia e Planejamento Ltda.

Dias, S. F. 1991. Estudo Ambiental no Utinga: vida útil do sistema de abastecimento d'água de Belém. Belém. IDESP. (Relatórios de Pesquisa, 19).

Grönblad, R. 1945. De Algis Brasiliensibus; Praecipue Desmidiaceis, in regione inferiore fluminis Amazonas a Prof. August Ginzberger (Wien) anno MCMXXVII collectis. Acta Societatis Scientiarum Fennicae, Nova serie B 2(6): 1-43.

Hegewald, E.; Hindák, F. \& Schnepf, E. 1990. Studies on the genus Scenedesmus Meyen (Chlorophyceae, Chlorococcales) from South India, with special reference to the cell wall ultrastructure. Nova Hedwigia 99: 1-73, pl.1-135.

Hindák, F. 1984. Studies on the Chlorococcal algae (Chlorophyceae) III. Bratislava, Veda, Slovak Academy of Sciences, V. (Biologické Práce, 1).

Hindák, F. 1990. Studies on the Chlorococcal algae (Chlorophyceae). V. Bratislava: Veda, Slovak Academy of Sciences. (Biologické Práce, v. 36).

Huszar, V.L. 1994. Fitoplâncton de um lago amazônico impactado por rejeito de bauxita (Pará, Brasil): estrutura da comunidade, flutuações espaciais e temporais. São Carlos. Universidade Federal de São Carlos. Tese de Doutorado.

IDESP. 1979. Projeto: Reconhecimento dos Recursos Naturais da Região Metropolitana de Belém. Belém. Coordenadoria de Pesquisa de Recursos Naturais.

Kammerer, G. 1938. Volvocalen und Protococcalen aus dem unteren Amazonasgebiet.. Sitzungsberichten der akademie der Wissenschaften in Wien Mathen-naturw, 147(5/10):183-228. 
Komárek, J. 1974. The morphology and taxonomy of crucigenoid algae (Scenedesmacede, Chlorococcales). Archiv fur Protistenkunde 116: 1-75.

Komárek, J. 1983. Contribution to the Chlorococcal Algae of Cuba. Hedwigia 37:65-180.

Komárek, J \& Fott, B. 1983. Chlorophyceae (Grünalgen) Ordnung: Chlorococcales. Die Binnengenasser 16: 1-1044.

Komárek, J. \& Ruzicka, J. 1969. Effect of temperature on the growth and variability os Scenedesmus quadricauda (Turp.) Breb. In: B. Fott (Ed.) Studies in Phycology. Stuttgart. E. Schweizerbart'ache Verlagsbuchandlung.

Köppen, W. 1948. Climatologia, con un estudio de los climas de la tierra. México. Fondo de Cultura Econômica.

Martins-da-Silva, R.C.V. 1994. Chlorophyceae (Algae, Chlorophyta) do lago Água Preta, município de Belém, Estado do Pará. Belém. Universidade Federal do Pará/Museu Paraense Emilio Goeldi. Dissertação de Mestrado.

Moreira, E. 1966. Belém e suas expressões geográficas. Belém. Imprensa Universitária.

Nogueira, I. S. 1991 Chlorococcales sensu lato (Chlorophyceae) do Município do Rio de Janeiro e arredores, Brasil: inventário e considerações taxonômicas. Rio de Janeiro. Universidade Federal do Rio de Janeiro. Dissertação de Mestrado.

Paiva, R.S. 1991. Composição e biomassa do fitoplâncton da Baía de Guajará (Pará-Brasil). Recife. Universidade Federal de Pernambuco. Dissertação de Mestrado.

RADAMBRASIL. 1976. Ministério das Minas e Energia. Departamento Nacional de Produção Mineral. Projeto RADAMBRASIL. Levantamento de Recursos Naturais, V.I.

Sant'Anna, C.L. 1984. Chlorococcales (Chlorophyceae) do Estado de São Paulo, Brasil. Vaduz. J. Cramer.

Sant'Anna, C.L.\& Martins, D.V. 1982. Chlorococcales (Chlorophyceae) dos lagos Cristalino e São Sebastião, Amazonas, Brasil: taxonomia e aspectos limnológicos. Revista Brasileira de Botânica 5: 67-82.

SECTAM. 1992. Parque Estadual do Utinga-Estudo Ambiental. Belém: Relatório Técnico SOF-REL-018/ 92

Stearn, W.T. 1983. Botanical Latin. History, Grammar, Syntax, Terminology and Vocabulary. David \& Charles.

Tell, G. \& Mosto, P. 1982. Flora Criptogámica de Tierra del Fuego: Chlorophyceae, Chlorococcales. Buenos Aires. Fundação para La Educacion, La Ciência y La Cultura.

Thomasson, K. 1971. Amazonian Algae. Mémoires de l'Intitut Royal des Sciences naturelles de Belgique 2(86):1-127.

Thomasson, K. 1977. Two conspicuous desmids from Amazonas. Botiska Notiser 130: 41-51.

Uherkovich, G. 1966. Die Scenedesmus-Arten ungarns. Budapest. Akadémiaikiadó. 173p.

Uherkovich, G. 1976. Algen aus den Flüssen Rio Negro und Rio Tapajós. Amazoniana 5(4): 465-515.

Uherkovich, G. 1981. Algen aus einigen Gewassern Amazoniens. Amazoniana 7(2): 191-219. 